\title{
Calibration parameter drift compensation of metal resistive bolometers operating in a thermal varying environment
}

\author{
F. Penzel ${ }^{\mathrm{a}}$, H. Meister ${ }^{\mathrm{a}}$, L. Giannone ${ }^{\mathrm{a}}$, D. Zhang ${ }^{\mathrm{b}}$ \\ ${ }^{a}$ Max Planck Institute for Plasma Physics, Bolzmannstr .2, 85748 Garching, Germany \\ ${ }^{b}$ Max Planck Institute for Plasma Physics, Wendelsteinstr. 1, 17491 Greifswald, Germany
}

\begin{abstract}
The ITER bolometer diagnostic will have to provide accurate measurements of the plasma radiation in a varying thermal environment of up to $250^{\circ} \mathrm{C}$. Current fusion experiments perform regular in-situ calibration of the sensor properties, assuming stable calibration parameters within short discharge times, e.g. $10 \mathrm{~s}$ on ASDEX Upgrade. For long-pulse fusion experiments, e.g. W7-X, the diagnostic is operated with water cooling for achieving a stable temperature environment. However, ITER will be equipped with about seventy bolometer cameras and is planned to have discharge times of up to $1 \mathrm{~h}$. Due to space restrictions, active cooling is not available for all locations. Thus, an alternative approach is required to allow for compensation of the changing calibration values due to thermal drifts. This paper demonstrates a method using the Wheatstone bridge current of the sensor to calculate in real-time the changing calibration values, such as the heat capacity and the thermal time constant. It is shown, that the thermal calibration parameter drift can be calculated by either extrapolating from the initial values or using a previously determined look-up table. Measurements in the ITER bolometer vacuum test facility (IBOVAC), used to simulate ITER-relevant thermal and vacuum environment, show that the change of the calibration values can be predicted during repeated thermal cycles over a duration sufficient for ITER discharges and even longer. Confidence intervals for the typical in-situ calibration method are determined and compared with the proposed extrapolation and look-up method for ITER, showing that these methods provide an equivalent quality of the measurement results.
\end{abstract}

Keywords: ITER, bolometer, calibration parameter drift compensation, thermal offset error compensation, IBOVAC

\section{Introduction}

The metal resistive bolometer is the most commonly used concept in tokamak and stellarator fusion experiments to provide spatially resolved measurements of the radiated power from the plasma. The sensors are based on a metallic and ceramic microstructure which is used as a broadband radiation absorber and temperature transducer [1]. The change of resistance of an integrated Wheatstone bridge is measured and used to calculate the absorbed power of the sensor.

The sensor is described as a thermal first-order linear time-invariant (LTI) system characterized by its thermal time constant $\tau$ and its heat capacity $\kappa$ [2]. These two values are then referred to as the calibration values of the sensor. They can be measured by heating the sensor with a known power and observing its temperature

Email address: florian.penzel@ipp.mpg.de (F. Penzel) step-response shortly before the discharge phase. However, the calibration values are only valid for a certain temperature range because they depend upon the thermal conductivity and the thermal contact conductance which again depend on the ambient temperature of the sensor.

As a solution for this, long-pulse fusion experiments with significant heating systems, e.g. W7-X, are operating the bolometer diagnostic in vessel ports with water cooling [3] in order to achieve a stable temperature environment. ITER will be equipped with about seventy bolometer cameras. They will be distributed all around the vacuum vessel (VV), the divertor, two upper and one equatorial port. Due to space restrictions, in particular with the bolometer cameras mounted onto the VV, active cooling is not available for all locations. And exactly these cameras are considered particularly relevant for physics studies and used to monitor and improve the massive gas injections of the Disruption Mitigation System ensuring the safe operation of ITER. Implementing 
active cooling for a diagnostic inside a nuclear facility such as ITER will be challenging and costly, consequently should be avoided for other cameras as well.

Theoretical work undertaken in the past, demonstrated the relationship between the offset voltage and the bridge current being a function of the ambient sensor temperature [4]. In this paper, the concept is extended towards the thermal drift of the calibration values ( $\kappa$ and $\tau)$. It is shown in an experimental setup, that the drift can be calculated in real-time eliminating this source of error for the calculation of the radiated power. Confidence intervals for the typical in-situ calibration method are determined and compared with the accuracy of the proposed extrapolation method for ITER showing that this method provides an equivalent quality of the measurement results.

\section{Experimental set-up}

The ITER bolometer vacuum test facility (IBOVAC), shown as a CAD image in figure 1, is used to examine the change of calibration parameters in a ITER-like thermal and vacuum environment.

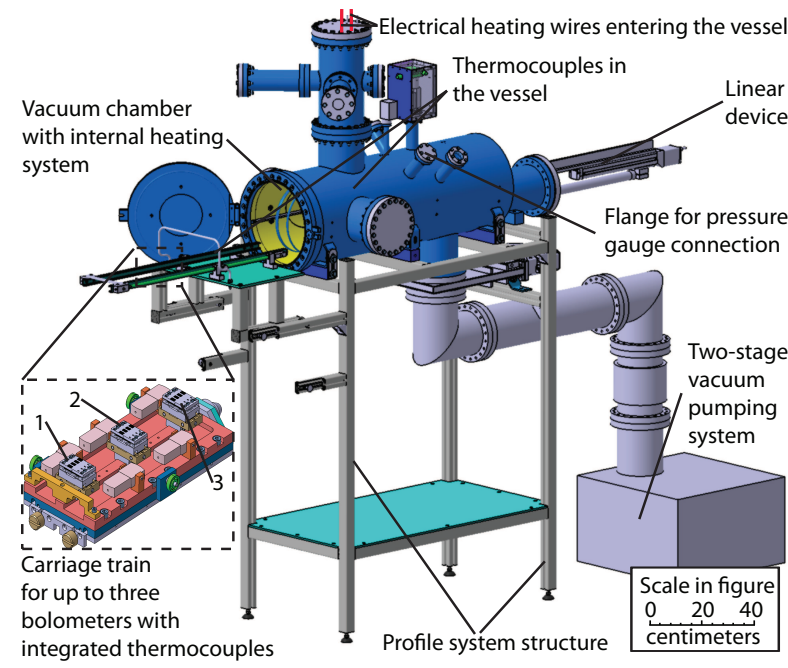

Figure 1: Perspective CAD image of the IBOVAC test facility with a description of the relevant components.

Up to three (four-channel) bolometer sensors can be mounted on a carriage train, electrically contacted and then inserted in the center of the vessel controlled by a linear device. The vessel can be heated through thermal radiation from a heating drum with coils around the carriage train (operated at $230 \mathrm{~V}, 11 \mathrm{~A}$ ) and through thermal conduction by a second heating circuit in the carriage train (operated at $24 \mathrm{~V}, 11 \mathrm{~A}$ ). Both heating circuits are feedback controlled with an on/off controller having a deadband of $1{ }^{\circ} \mathrm{C}$. Thermocouples of type $\mathrm{K}$ are distributed on several positions in the vessel including one being integrated in each one of the bolometer sensor holders to be as close as possible to the sensor itself. Internal temperatures of up to $450{ }^{\circ} \mathrm{C}$ can be reached and permanently kept in order to simulate ITER-like bake out temperature cycles or other needed temperature profiles. The outer surface of the heating drum and the inner surface of the vacuum-vessel are fitted with thermal shields to reduce heat transport by radiation. An additional thermal insulation on the outside of the vacuum-vessel reduces energy loss. The vacuum vessel is pumped in two stages by the dry roughing pump Adixen ACP28 and the turbo pump Adixen ATP400 enabling operation at pressures down to $10^{-8} \mathrm{mbar}$ at $25^{\circ} \mathrm{C}$. The pressure inside IBOVAC is monitored using the compact full range gauge Pfeiffer PKR 251 and the baratron MKS type 120A. Temperature and pressure values can be acquired with a sample rate of up to $10 \mathrm{~Hz}$.

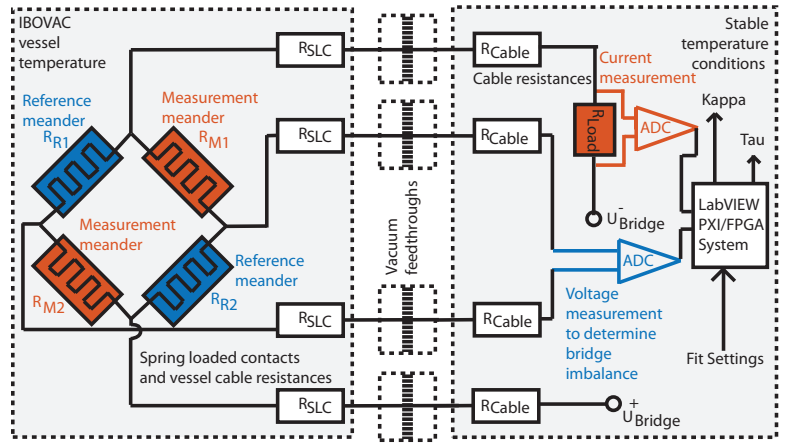

Figure 2: Simplified equivalent circuit of IBOVAC showing the relevant temperature dependent resistances.

Control and data acquisition of all components of the test facility IBOVAC is done using a dedicated software programmed in National Instruments LabVIEW, runnning on a PXI/FPGA system. The same calibration and measurement procedures in this software are currently in use at ASDEX Upgrade and W7-X bolometer systems [5]. This has the advantage that the methods shown in this paper will be easy to implement on these devices.

Figure 2 shows the equivalent circuit (EC) of IBOVAC containing the relevant resistances: The reference meander resistances $\left(R_{R 1}, R_{R 2}\right)$, the measurement meander resistances $\left(R_{M 1}, R_{M 2}\right)$, the resistances due to the spring loaded contacts, the in vessel cable resistances $\left(4 x R_{S L C}\right)$ and the external cable resistances $\left(4 x R_{\text {Cable }}\right)$. Simplified symbols for the analog to digital converters (ADC) performing the current and voltage measurement 
of the bridge are shown as well. The special characteristic of the system here, is that each channel contains an additional ADC with load resistor allowing to measure the current through the entire bridge circuit. Intention of this EC is to show also which resistances are part of the varying thermal environment respectively which ones have to be taken into account when calculating the impact on the total current through the system.

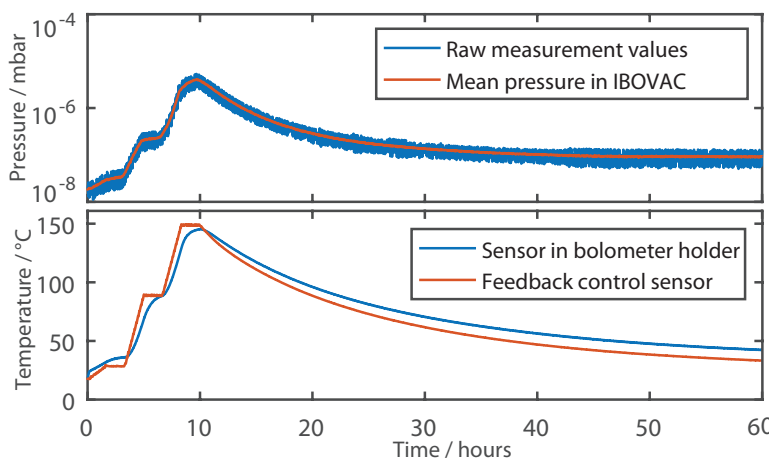

Figure 3: Temperature and pressure of the reference scenario used for the investigation of the parameter dependencies, captured at $0.1 \mathrm{~Hz}$

Figure 3 shows the thermal and pressure reference scenario (RSC) used for all results shown in this paper. The vacuum vessel is heated in three stages, $30^{\circ} \mathrm{C}$, $90^{\circ} \mathrm{C}, 150^{\circ} \mathrm{C}$ with a heat ramp of $1{ }^{\circ} \mathrm{C} / \mathrm{min}$. Then, it is left to cool down for about 2 days. Operation at $>150{ }^{\circ} \mathrm{C}$ are possible but would exceed the specifications of the spring loaded contacts which electrically connect the bolometer sensors and could add uncertainties to the interpretation of the signals. Thus, this is avoided here, in particular for an experiment which focuses on the question of measurement quality. At the beginning of the run, the pressure is at the lowest achievable value of the pumping system. Due to the internal heating the pressure rises, but stays always $<10^{-5}$ mbar. Therefore, heat transfer via conduction and convection by the presence of surrounding gas is effectively eliminated.

\section{Uncertainty of typical calibration method}

The experiments have been performed with two different $\mathrm{Si}_{3} \mathrm{~N}_{4}$ based bolometer sensors manufactured by the Fraunhofer ICT-IMM with Au absorbers and Pt meanders. Bolometer $2^{1}$ has a $12 \mu \mathrm{m}$ absorber and $3 \mu \mathrm{m}$ membrane, bolometer $3^{2}$ has a $6 \mu \mathrm{m}$ absorber and $5 \mu \mathrm{m}$

${ }^{1}$ Fraunhofer IMM-ICT Flow-Chart 1100025, Wafer 481, Chip E ${ }^{2}$ Fraunhofer IMM-ICT Flow-Chart 900077, Wafer 181, Chip D membrane. Both sensors are coated with $150 \mathrm{~nm} \mathrm{Al}$ on the absorber side to enhance the heat conductivity.
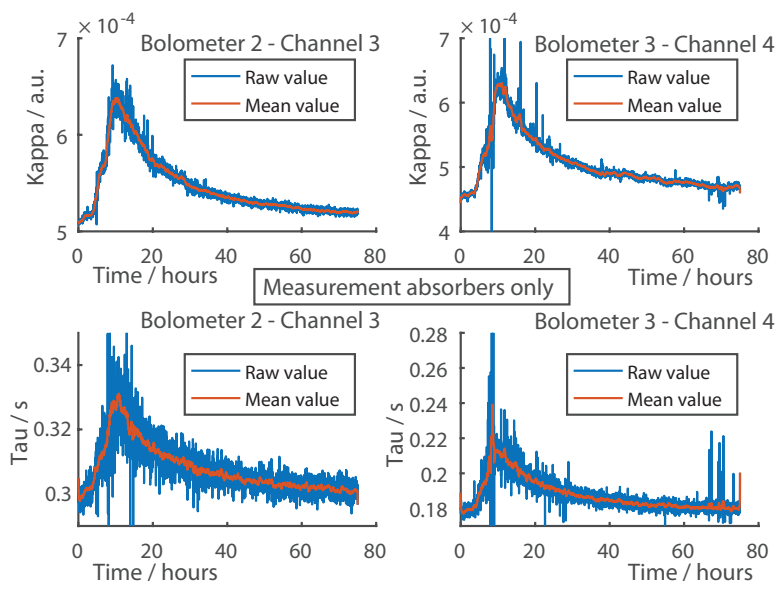

Figure 4: $\tau$ and $\kappa$ raw and mean value during the typical calibration procedure showing the measurement uncertainties.

Figure 4 shows the two calibration values $\tau$ and $\kappa$ (given in normalized values according to [2]) for the measurement absorber of each sensor. The raw measurement values show a significant dispersion compared to the mean value calculated with a moving average of 20 samples. These deviations can be used as a measure for the relative uncertainties of the typical calibration procedure and are most likely to be attributed to the performance of the used electronics. Bolometer 2 has a twice as thick absorber and a $40 \%$ thinner membrane than bolometer 3 which result in a larger time constant $\tau$ and heat capacity $\kappa$. The relative uncertainties are similar for both bolometer types, for $\kappa_{\Delta}=2-5 \%$ and for $\tau_{\Delta}=3-6 \% . \kappa$ is significantly affected by a change of ambient temperature: A rate of increase of $12.8 \frac{a . u \text {. }}{K}$ $(0.16 \% / \mathrm{K})$ can be calculated from the measurements in IBOVAC. $\tau$ is generally less affected, the rate of increase is $0.24 \frac{m s}{K}(0.08 \% / \mathrm{K})$. However, the rate of increase of $\tau$ is twice as high as for the previously used $\mathrm{Au} / \mathrm{Kapton}$ based bolometers [2].
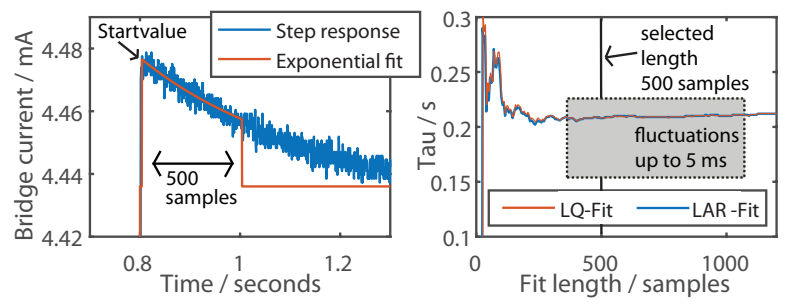

Figure 5: Left: Current step response and exponential fit to determine $\tau$. Right: Calculated $\tau$ value over different fit lengths. 
Systematic errors which depend on the method of determining the calibration parameters should also be taken into account. Therefore, the influence of the fit choice and its settings used to calculate $\tau$ and $\kappa$ has been examined. Figure 5 shows on the left the step response of the bolometer sensor with its exponential fit in order to determine the time constant of the LTI system.

The plot on the right shows the resulting value plotted over different fit lengths (\# measured samples from the decay) and for two typically used fit types. It can be seen that the final value of $\tau$ fluctuates up to $5 \mathrm{~ms}$ which corresponds to a difference of $2.5 \%$. With short fit lengths $(\#<300) \tau$ varies quite a lot and with too large fit lengths (\# > 500) noise starts to increase the fit result. A shifting of the start value, which corresponds in selecting the sample area to be used, and the fit methods Least Square (LQ), Least Absolute Residual (LAR) and Bisquare (not shown) have been compared as well. Deviations in the plot are barely visible, they result in $\tau_{\text {Fit-Met }}=0.5 \%$ corresponding to $\approx 1 \mathrm{~ms}$. For the following analysis it was decided to use an LQ fit with $\#=500$ acquired at $2.5 \mathrm{kHz}$.

\section{Extrapolation and look-up method for drift com- pensation}

The principle of the proposed compensation methods is to determine before the discharge the bolometer calibration parameters as a function of the temperature. During the discharge and the normal operation of the diagnostic, a real-time temperature drift compensated value can then be calculated. As an alternative, a previously determined look-up table acquired during vessel bakeout or in a test facility such as IBOVAC can be used. Both methods will be compared at the end of this section. Figure 6 shows in the top right image the bridge current plotted against time and on the top left image the bridge current against the bolometer holder temperature during the RSC for the same channels as shown in Section 3. The bridge current decreases with higher temperatures as expected from the positive temperature coefficient (TCR) of the Pt meander. Bolometer 2 - Channel 3 shows a small hysteresis for temperatures above $130^{\circ} \mathrm{C}$ which is due to the erratic nature of the spring loaded contacts operated at high temperatures and could be seen from time to time on other measurements as well. But the measured current values are generally stable and very low-noise, thus will be well-suited as input for extrapolations.

The Wheatstone bridge is operated with $U_{\text {Bridge }}=10 \mathrm{~V}$ and the resistance of the bridge components are $R_{M 1, M 2, R 1, R 2} \approx 1200 \Omega$ which results
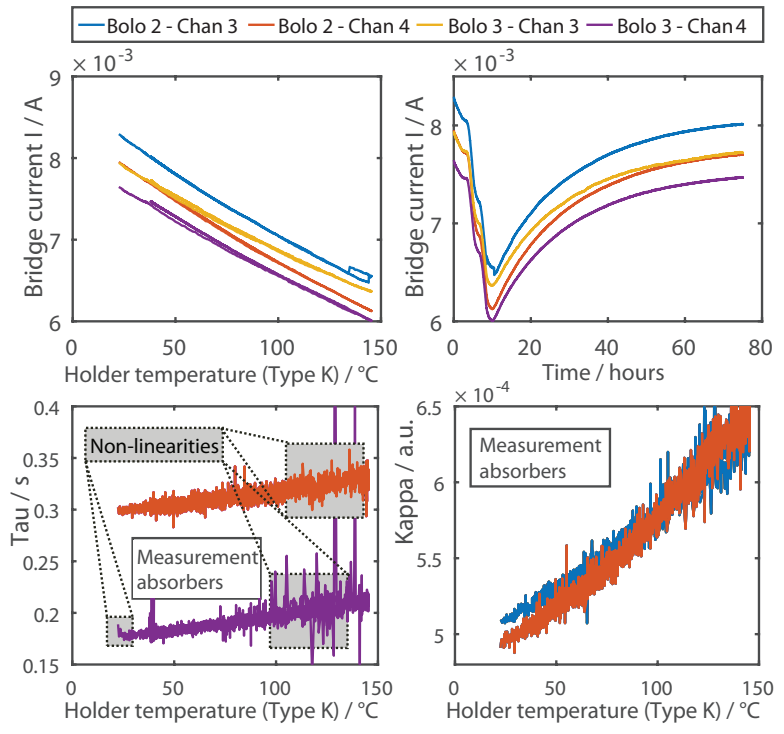

Figure 6: Top: Wheatstone bridge current plotted against the bolometer holder temperature and the time. Bottom: $\tau$ and $\kappa$ plotted against the bolometer holder temperature.

in a $I_{\text {Bridge }} \approx 8 \mathrm{~mA}$ thus confirming that the values are correctly acquired. The simplest approach is to assume linearity during the temperature ramp $\Delta T=125 \mathrm{~K}$ of the RSC. Thus, a TCR of $k_{T_{0}^{\text {IBOVAC }}}=2.1 \mathrm{mK}^{-1}$ can be derived for a median reference temperature of $T_{0}^{I B O V A C}=62.5^{\circ} \mathrm{C}$. The TCR corresponds to data provided from the bolometer manufacturer Frauenhofer ICT-IMM $\left(k_{T_{0}^{I M M}}=1.9-2.1 \mathrm{mK}^{-1}\right)$ measured at a median temperature of $T_{0}^{I M M}=55^{\circ} \mathrm{C}$.

The two plots in the bottom of figure 6 show the calibration values as a function of the bolometer holder temperature. Data from two different measurement absorber channels, from Bolometer 2 and 3 are shown: On the left, the values for $\tau$ and on the right the values for $\kappa . \kappa$ shows a slight exponential shape besides that both channels shown here have a different gradient. $\tau$ shows averaged over the whole RSC a more linear behavior and a temperature dependency roughly half the rate of $\kappa$. However, certain non-linear fluctuations, here marked with grey boxes at the beginning of the RSC and for higher temperatures would make a linear approximation imprecise. The differences between each channel can most probably be associated with production tolerances of the thin film manufacturing process. Moreover, irregularities during the bolometer holder assembly such as applying different contact pressures on the bolometer sensor holder subcomponents could have an effect on the overall thermal conductivity of the system. In conclusion, each channel will have to be asso- 
ciated with different drift compensation factors and will need its independent assessment.
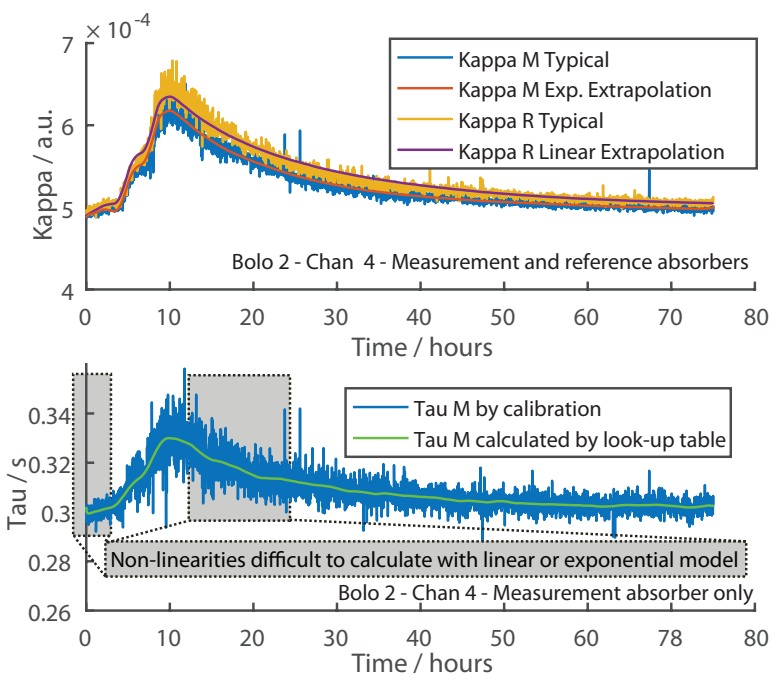

Figure 7: Extrapolation of $\kappa$ through the bridge current as a measure of the bolometer temperature. $\tau$ is calculated using a look-up table.

Figure 7 shows two plots for $\kappa$ and $\tau$ values for Bolometer 3 acquired with the typical calibration procedure and compared to the drift compensated values. For $\kappa$, the data of measurement $\left(\kappa_{M}\right)$ and reference absorber $\left(\kappa_{R}\right)$ has been used. For $\tau$, only the data of the measurement absorber. Three extrapolation methods are compared to the measurement values: $\kappa_{R}$ is calculated with a linear extrapolation ${ }^{3}$ and $\kappa_{M}$ is calculated with an exponential extrapolation ${ }^{4}$ and $\tau_{M}$ is derived by using a look-up table. The look-up table is based on the measurement and averaging of 3 consecutive RSC's. All methods are within the uncertainty of the typical calibration procedure $\left(\kappa_{\Delta}<2-5 \%, \tau_{\Delta}<3-6 \%\right)$ and can reproduce the parameter drift with sufficient precision. The look-up table performs generally better, because the linear or exponential function have difficulties to model nonlinearities or discontinuities of the parameter course (marked with grey boxes). However, it takes more effort to set up this method.

\section{Discussion and further work}

It has been shown that the change of the calibration values can be predicted during thermal cycles for periods longer than necessary for the ITER discharges.

\footnotetext{
${ }^{3} \kappa_{R}=a_{R 07} * I_{\text {bridge }}+b_{R 07}, a_{R 07}=-0.082, b_{R 07}=0.0011$

${ }^{4} \kappa_{M}=a_{M 07} * \exp \left(b_{M 07} * I_{\text {bridge }}\right), a_{M 07}=0.00136, b_{M 07}=-131.3$
}

Measurement uncertainties for the typical in-situ calibration method have been determined and are compared with the accuracy of the proposed extrapolation and look-up method. Both methods provide sufficient accuracy of the calibration values $\tau$ and $\kappa$ compared to the currently systematic uncertainties introduced by the electronics setup. Maximum operation temperatures of the bolometer in ITER are currently estimated to be around $250{ }^{\circ} \mathrm{C}$. Assuming a continuous parameter drift up to these high temperatures $(\Delta T=225 K), \tau$ would deviate $18 \%$ and $\kappa$ up to $36 \%$ of its initial value at room temperature $\left(T=25^{\circ} \mathrm{C}\right)$. Therefore, it will be crucial to implement a method of drift compensation for the ITER bolometer sensors in order to provide reliable and precise measurement data. Thus, in order to validate the methods for higher temperatures a bonded connection of the sensor should be envisaged, which for the operation in ITER will be necessary anyway. Moreover, it is likely that the non-linear profile of $\tau$ will disappear with a more stable electric connection.

In ITER, the calibration parameter dependencies should be determined periodically during bake out. Due to neutron-induced degradation and transmutation of sensor materials such as Au it likely that these dependencies will drift over the ITER lifetime anyway. Another aspect to be considered for ITER is the influence of the internal $\mathrm{VV}$ cable resistance $\left(R_{S L C}\right.$ in the EC of IBOVAC). For short experiments, the temperature (and resistance) increase of the bolometer sensor and holder will not correspond to the temperature (and resistance) increase of the VV cable during bake out. Thus, the predetermined parameter dependencies as a function of $f_{\tau, \kappa}\left(4 * R_{S L C}+R_{M 1, M 2, R 1, R 2}\right)$ could deviate. This deviation can be calculated by a correction factor (CF) given by the ratio of the relevant resistances and their TCR's: $C F=\frac{4 * R_{S L C}}{R_{M 1, M 2, R 1, R 2}} * \frac{k^{R} S L C}{k^{B r i d g e R}}$. In case the resistance values of the final selected VV MI-cables remain below $300 \mathrm{~m} \Omega / \mathrm{m}$ this results in an effect of $C F \approx 1 \%{ }^{4}$, which can be neglected.

Alternatively to the methods suggested, one could think about implementing a blind channel into each bolometer camera. Reference and measurement sensor of this channel would be completely shielded from radiation allowing to constantly determine any change of calibration values related to the change of the environment. Differences between each individual sensors and deviations due to manufacturing tolerances can not be resolved with this method. But for detecting fast events,

\footnotetext{
${ }^{4} R_{S L C}=5 \mathrm{~m} * 300 \mathrm{~m} \Omega / \mathrm{m}, R_{M 1, M 2, R 1, R 2}=1.2 \mathrm{k} \Omega, \frac{k^{R} S L C}{k^{\text {BridgeR }}}=2 / 1$, $\mathrm{Cu}$-cables $\left(k^{R_{S L C}}=4 \mathrm{mK}^{-1}\right)$ and Pt-sensor $\left(k^{\text {Bridge } R}=2 \mathrm{mK}^{-1}\right)$
} 
such as pressure changes this might be an alternative approach. However, space for additional electrical connections is not available at all bolometer locations in ITER so this should only be considered as a complementing method for cameras with sufficient space such as port plug cameras.

Finally, it must be noted that the calibration values are a potential source of error when determining the measured power in a fusion experiment because they will always have a limited accuracy. For bolometer systems using the same electronics and software setup as in IBOVAC, $\kappa$ and $\tau$ have a total uncertainty between $3-9 \%$ depending if using multiple channels ( $3 \%$ uncertainty) during the data evaluation and errors are uniformly distributed or when individual channels are evaluated ( $9 \%$ uncertainty). The impact on resulting parameters such as the total radiated power of the plasma $P_{\text {rad }}$ has to be assessed as a whole because its uncertainty will depend on the used method (e.g. explicit tomographic method and its boundary conditions) and other quantities such the inaccuracies of the LOS characteristics[6] and its alignment[7].

\section{Acknowledgments}

The authors acknowledge the accurate review of A. Kampke and C. Rapson.

\section{References}

[1] K. F. Mast, J. C. Vallet, C. Andelfinger, P. Betzler, H. Kraus, G. Schramm, A low noise highly integrated bolometer array for absolute measurement of VUV and soft-X radiation, Review of Scientific Instruments 62 (3) (1991) 744-750.

[2] L. Giannone, K. Mast, M. Schubert, Derivation of bolometer equations relevant to operation in fusion experiments, Review of Scientific Instruments 73 (9) (2002) 3205-3214.

[3] D. Zhang, R. Burhenn, R. Koenig, L. Giannone, P. Grodzki, B. Klein, K. Grosser, J. Baldzuhn, K. Ewert, V. Erckmann, Design criteria of the bolometer diagnostic for steady-state operation of the W7-X stellarator, Review of Scientific Instruments 81 (10) (2010) 10E134-10E134.

[4] D. Zhang, H. M. Gianonne, R. Knig, R. Burhenn, Thermal drift study on the bolometer diagnostic for steady-state fusion plasmas, 39th EPS Conference on Plasma Physics and 16th International Congress on Plasma Physics - P5.040.

[5] L. Giannone, T. Eich, J. Fuchs, M. Ravindran, Q. Ruan, L. Wenzel, M. Cerna, S. Concezzi, Data acquisition and real-time bolometer tomography using LabVIEW RT, Fusion Engineering and Design 86 (6-8) (2011) 1129 - 1132. doi:10.1016/j.fusengdes.2011.03.119.

[6] F. Penzel, H. Meister, L. Giannone, M. Kannamüller, J. Koll, T. Trautmann, A. W. Koch, Assessment of line of sight characteristics of ITER bolometer prototype collimators, Fusion Engineering and DesignProceedings of the 27th Symposium of Fusion Technology (SOFT-27).
[7] F. Penzel, H. Meister, M. Bernert, T. Sehmer, T. Trautmann, M. Kannamüller, J. Koll, A. Koch, Automated in situ line of sight calibration of ASDEX Upgrade bolometers, Fusion Engineering and Design, Proceedings of the 11th International Symposium on Fusion Nuclear Technology-11 (ISFNT-11) 89 (9) (2014) 22622267. 